\title{
Effects of early nutrition and growth on brain volumes, white matter microstructure, and neurodevelopmental outcome in preterm newborns
}

\author{
Caterina Coviello ${ }^{1}$, Kristin Keunen ${ }^{1}$, Karina J Kersbergen ${ }^{1}$, Floris Groenendaal ${ }^{1}$, Alexander Leemans ${ }^{2}$, Barbara Peels ${ }^{1}$, \\ Ivana Isgum ${ }^{2}$, Max A Viergever ${ }^{2}$, Linda S de Vries ${ }^{1}$, Giuseppe Buonocore ${ }^{3}$, Virgilio P Carnielli ${ }^{4}$ and Manon J N L Benders ${ }^{1}$
}

BACKGROUND: This study aimed to investigate the effect of nutrition and growth during the first 4 weeks after birth on cerebral volumes and white matter maturation at term equivalent age (TEA) and on neurodevelopmental outcome at 2 years' corrected age (CA), in preterm infants.

METHODS: One hundred thirty-one infants born at a gestational age $(G A)<31$ weeks with magnetic resonance imaging (MRI) at TEA were studied. Cortical gray matter (CGM) volumes, basal ganglia and thalami (BGT) volumes, cerebellar volumes, and total brain volume (TBV) were computed. Fractional anisotropy (FA) in the posterior limb of internal capsule (PLIC) was obtained. Cognitive and motor scores were assessed at 2 years' CA.

RESULTS: Cumulative fat and enteral intakes were positively related to larger cerebellar and BGT volumes. Weight gain was associated with larger cerebellar, BGT, and CGM volume. Cumulative fat and caloric intake, and enteral intakes were positively associated with FA in the PLIC. Cumulative protein intake was positively associated with higher cognitive and motor scores (all $P<0.05$ ).

CONCLUSION: Our study demonstrated a positive association between nutrition, weight gain, and brain volumes. Moreover, we found a positive relationship between nutrition, white matter maturation at TEA, and neurodevelopment in infancy. These findings emphasize the importance of growth and nutrition with a balanced protein, fat, and caloric content for brain development.

$\mathbf{P}$ ostnatal growth failure is a common consequence of very preterm birth. A large proportion of very-low-birthweight infants is growth-restricted by the time of hospital discharge and these growth deficits have been reported to persist into childhood and adolescence $(1,2)$. Common causes of postnatal growth failure are early postnatal malnutrition as a consequence of feeding intolerance and high levels of energy expenditure because of neonatal illness (e.g., postnatal infections and need for mechanical ventilation). In the first days after birth, preterm infants depend on parenteral nutrition (PN) as their primary source of calories and nutrients, which renders them vulnerable to macro- and micronutrient deficits $(2,3)$.

The developing brain is particularly susceptible to postnatal growth deficits, because crucial processes of brain growth and maturation take place in the preterm period (i.e., $24-40$ weeks' postmenstrual age, PMA) (4). The cerebellum and cortical gray matter exhibit the highest growth rates, with four- to fivefold increases in volume $(5,6)$. Moreover, the cortex undergoes a remarkable transformation from a largely lissencephalic structure at the end of the second trimester to a highly convoluted mantle at around term equivalent age (TEA) that approximates the morphology of the adult cerebral cortex (5). Concomitantly, axons develop in the white matter and form connections, neurons migrate from the subplate to the cortical plate, and pre-oligodendrocytes mature to myelin-producing mature oligodendrocytes (7). Thus far, little is known about the impact of early postnatal growth and nutrition in preterm infants on brain development. Vinall et al. demonstrated slower body growth between birth and TEA to be related to higher fractional anisotropy (FA) in the cortex, reflecting delayed cortical maturation (8). Although nutritional intake was not included in their analyses, findings of this study suggest a pivotal role for the latter. A recent paper showed that impaired caloric, protein and fat intake during the first 2 weeks after birth were related to dysmaturation of the cortex, deep grey matter, and cerebellum, evaluated at TEA (9). Tan et al. reported a correlation between energy deficit during the first month after birth and total brain volume (TBV) at TEA, and between protein-energy deficit and neurodevelopmental outcome at 3 months post-term in infants born before 29 weeks' gestation (10). Another study in 51 preterm infants did not find a significant relationship between caloric and protein intake and brain volumes, although this study was not powered to assess this association (11). Moreover, research

\footnotetext{
'Department of Neonatology, Wilhelmina Children's Hospital, University Medical Center Utrecht and Utrecht University, Utrecht, The Netherlands; ${ }^{2}$ Image Sciences Institute, University Medical Center Utrecht and Utrecht University, Utrecht, The Netherlands; ${ }^{3}$ Department of Pediatrics, Siena University, Siena, Italy: ${ }^{4}$ Department of Neonatology, Polytechnic University of Marche and Salesi Children's Hospital, Ancona, Italy. Correspondence: Manon J.N.L. Benders (m.benders@umcutrecht.nl) 
has shown that nutritional components might influence gut microbiota and this, in turn, may have an impact on brain development and plasticity, through immunological, endocrine, and neural pathways (12). Hence, the role of postnatal nutrition and body growth in brain development needs to be further investigated. We therefore aimed to extend the findings from previous observations and investigate the association between early postnatal nutritional intake and postnatal growth on brain volumes of specific brain structures and white matter maturation in very preterm infants at TEA, and on neurodevelopmental outcome at 2 years' corrected age (CA). We hypothesized that protein and caloric intake would be related to larger brain volumes of the cerebellum, cortex, and central gray matter, and to improved white matter maturation, reflected by higher FA in the posterior limb of the internal capsule (PLIC) in preterm infants. Furthermore, we postulated that nutritional intake would be associated with higher cognitive and motor scores in late infancy.

\section{METHODS}

\section{Subjects}

Preterm infants with a gestational age (GA) below 31 weeks, admitted to the level III neonatal intensive care Unit of the Wilhelmina Children's Hospital (Utrecht, The Netherlands) between January 2007 and July 2012, for whom details about nutritional intake were available for at least 28 days after birth and an magnetic resonance imaging (MRI) had been successfully acquired at TEA, were included in this study. Infants with congenital malformations, inborn errors of metabolism, and/or congenital infections of the central nervous system were excluded. The Institutional Review Board of the University Medical Center Utrecht granted ethical permission. Written informed consent was obtained from the parents.

\section{Clinical Parameters}

Prenatal, perinatal, and postnatal data were collected by retrospective chart review and are outlined in Table 1 . In addition, weight gain, increase in head circumference (HC), length, days of PN, and time until full enteral feeding were extracted from the medical records. Weight gain was defined in $\mathrm{g} / \mathrm{kg} /$ day over the first 28 days and was calculated by exponential method as reported by Patel et al. (13). Increase in $\mathrm{HC}$ and length was calculated in $\mathrm{cm} /$ week over the first 4 weeks after birth.

\section{Nutritional Intake}

Data on daily cumulative parenteral and enteral protein, fat, and caloric intake during the first 28 days after birth were retrieved from the electronic medical records. Enteral nutrition from mother's milk (MM) and preterm infant formula (Friso Prematuur; FrieslandCampina Singapore, Singapore) was recorded separately. MM was assumed to contain $75 \mathrm{Kcal} / 100 \mathrm{ml}, 1.4 \mathrm{~g}$ of protein/100 ml, $3.4 \mathrm{~g}$ of fat $/ 100 \mathrm{ml}$, and $6.6 \mathrm{~g}$ of carbohydrate $/ 100 \mathrm{ml}$ (ref. 14). Macronutrient contents of formula, human milk fortifier, and parenteral solutions were calculated based on the published manufacturers' recordings and included protein, lipids, and glucose expressed in g/ $\mathrm{kg}$ administered over the first 28 days. Cumulative caloric intake was calculated based on the cumulative amount of parenteral and enteral nutrition and was calculated in $\mathrm{kcal} / \mathrm{kg}$ over the first 28 days.

\section{Feeding Protocol}

The nutrition protocol at our institution consisted of enteral and PN that was initiated as soon as possible after birth and was slowly increased to $150-180 \mathrm{ml} / \mathrm{kg} /$ day, which was aimed to be reached on day 7 after birth. Minimal enteral feeding was introduced within 24-
Table 1. Clinical characteristics of the study population

\begin{tabular}{|c|c|}
\hline $\begin{array}{l}\text { Clinical characteristics } N=131 \text { preterm } \\
\text { infants }\end{array}$ & $\begin{array}{l}\text { Mean and SD or } \\
N \text { (percentage) }\end{array}$ \\
\hline Gestational age (weeks) & $27.0 \pm 1.5$ \\
\hline Birth weight $(\mathrm{g})$ & $934 \pm 193$ \\
\hline Birth weight $Z$-score ${ }^{a}$ & $-0.18 \pm 0.82$ \\
\hline $\begin{array}{l}\text { Small for gestational age (birth weight } \\
\text { percentile }<10 \text { ) }\end{array}$ & $11(8 \%)$ \\
\hline Head circumference $Z$-score & $-0.1 \pm 0.97$ \\
\hline Males (no, \%) & $71(54 \%)$ \\
\hline Singleton (no, \%) & $102(78 \%)$ \\
\hline Mechanical ventilation (days) & $9 \pm 9$ \\
\hline Bronchopulmonary dysplasia ${ }^{\mathrm{b}}$ & $24(18 \%)$ \\
\hline $\mathrm{PDA}^{\mathrm{b}}$ & $60(46 \%)$ \\
\hline Sepsis ${ }^{c}$ & $50(38 \%)$ \\
\hline Necrotizing enterocolitis ${ }^{d}$ & $7(5 \%)$ \\
\hline Intraventricular hemorrhage grades I-II & $44(34 \%)$ \\
\hline Intraventricular hemorrhage grades III-IV & $5(4 \%)$ \\
\hline $\begin{array}{l}\text { Post-hemorrhagic ventricular dilatation } \\
\text { requiring intervention }\end{array}$ & $7(5 \%)$ \\
\hline Mild white matter injury score ${ }^{f}$ & $89(67 \%)$ \\
\hline Moderate-severe white matter injury score ${ }^{f}$ & $18(14 \%)$ \\
\hline Cerebellar hemorrhages & $15(11 \%)$ \\
\hline Postmenstrual age at scan (weeks) & $41.4 \pm 0.8$ \\
\hline
\end{tabular}

BPD, bronchopulmonary dysplasia; IVH, Intraventricular hemorrhage; MRI, magnetic resonance imaging; NEC, necrotizing enterocolitis; PDA, patent ductus arteriosus; PMA, postmenstrual age; PVHD, post-hemorrhagic ventricular dilatation; TEA, term equivalent age.

airth weight Z-score was computed according to the Dutch Perinatal registry reference data(31).

DPDA requiring drug treatment or surgical ligation. BPD was defined as supplemental oxygen requirement at 36 weeks' PMA.

'Sepsis was defined as blood culture-proven sepsis.

${ }^{d}$ NEC was defined as Bell's stage two or higher. IVH was classified according to the grading system proposed by Papile and colleagues ${ }^{41}$ and assessed on sequential cranial ultrasound and MRI at TEA.

eThe intervention in PVHD was defined as a neurosurgical intervention and/or lumbar punctures.

'White matter injury was evaluated according to the scoring system by Woodward et al. (15). Cerebellar hemorrhages were classified according to the cerebellar injury score reported by Kidokoro et al. (16).

$48 \mathrm{~h}$ after birth. The amount was increased by $20 \mathrm{ml} / \mathrm{kg}$ every day if enteral nutrition was tolerated. MM was enriched with fortifier when enteral feeding of $120 \mathrm{ml} / \mathrm{kg} /$ day was reached (Friso BMF, FrieslandCampina Singapore). Preterm formula was administered to the infant when MM was not available. PN was introduced on the first day after birth containing glucose and amino acids (Primene 10\%). A lipid solution (Intralipid 20\%) was added on day 2 after birth. Amino acid and lipid intake were slowly increased to a maximum of $2.6 \mathrm{~g} / \mathrm{kg}$ amino acids on day 3 or 4 after birth and $1.7 \mathrm{~g} / \mathrm{kg}$ lipids on day 4 or 5 after birth.

\section{Magnetic Resonance Imaging}

MR investigations were performed on a 3 tesla MR system (Philips Healthcare, Best, the Netherlands) using an eight-channel SENSE head coil. Infants were sedated using $50-60 \mathrm{mg} / \mathrm{kg}$ chloral hydrate administered by nasogastric tube $15 \mathrm{~min}$ before the examination as per the clinical protocol. Next, infants were placed in a vacuum fixation pillow to reduce spontaneous head movements (Kohlbrat and Bunz, Radstadt, Austria) and earmuffs (Natus Medical, San Carlos, 


\section{Articles $\mid$ Coviello et al.}

CA) were applied for hearing protection. Heart rate, transcutaneous oxygen saturation, and respiration rate were continuously monitored and a neonatologist was present throughout the entire examination. The scanning protocol included T2- and T1-weighted imaging and diffusion tensor imaging (DTI). Parameters of the scanning protocol included the following: axial three-dimensional (3D) T1-weighted image (Repetition time $(\mathrm{TR})=9.4 \mathrm{~ms}$; Echo time $(\mathrm{TE})=4.6 \mathrm{~ms}$; voxel size $0.94 \times 0.94 \times 2.0 \mathrm{~mm}$; no gap), coronal 3D T1-weighted image (TR 9.5 $=\mathrm{ms} ; \mathrm{TE}=4.6 \mathrm{~ms}$, voxel size $0.78 \times 0.91 \times 1.2 \mathrm{~mm}$; no gap), axial T2-weighted image $(\mathrm{TR}=6,293 \mathrm{~ms} ; \mathrm{TE}=120 \mathrm{~ms}$, voxel size $0.54 \times 0.61 \times 2.0 \mathrm{~mm}$; no gap), and coronal T2-weighted image $(\mathrm{TR}=4,847 \mathrm{~ms} ; \mathrm{TE}=150 \mathrm{~ms}$, voxel size $0.78 \times 0.89 \times 1.2 \mathrm{~mm}$; no gap). T2- and T1-weighted images were used to evaluate brain injury, defined as white matter injury (WMI) assessed according to the WMI score proposed by Woodward et al. (15) and cerebellar injury assessed according to Kidokoro et al. (16). Two neonatologists with extensive experience in the field of neonatal neuroimaging (LdV and MB) evaluated the MRI scans for brain injury. The DTI protocol included 32 weighted diffusion scans $\left(b=800 \mathrm{~s} / \mathrm{mm}^{2}\right)$ and one nonweighted scan $\left(b=0 \mathrm{~s} / \mathrm{mm}^{2}\right)$; single-shot echo planar imaging with 50 slices covering the entire brain ( $\mathrm{TR}=5,736 \mathrm{~ms}$; $\mathrm{TE}=70 \mathrm{~ms}$, voxel size $1.4 \times 1.4 \times 2.0 \mathrm{~mm}$; no gap).

\section{Volumetric Measurements}

Brain volumes were computed using an automatic segmentation method that segments eight different brain structures; cortical gray matter (CGM), basal ganglia, cerebral ventricles (Vent), unmyelinated white matter, myelinated white matter, brainstem, cerebellum, and TBV (17). From these segmentations, CGM, basal ganglia and thalami (BGT) volumes, and cerebellar volumes were selected for further analyses based on the a priori hypothesis that nutrition would particularly influence growth and development of these structures. TBV was defined as the volume of all brain structures, that is, intracranial volume without the volume of the ventricles and cerebrospinal fluid. A representative example of the automatic segmentation results is outlined in Figure 1.

\section{DTI Analysis}

All images were visually inspected to ensure good data quality. DTI images were first corrected for eddy current distortions and small head movements. Data sets were excluded in case five or more volumes (i.e., diffusion directions) had motion artifacts. Second, the diffusion tensor was fitted to each voxel using a reweighted linear least squares estimation after excluding outliers using a robust fitting approach (REKINDLE). An automated neonatal atlas (18) was registered to the FA maps of the DTI data using affine and elastic registration "Elastix" (19). Outliers were not manually excluded because the tensor reconstruction algorithm accounts for that. FA values of the bilateral PLIC were calculated based on the automated neonatal atlas. Representative examples of the registration of the PLIC from the neonatal atlas to individual FA maps are provided in Figure 2.

\section{Neurodevelopment Assessment}

Neurodevelopmental outcome was assessed at 2 years' CA using the Bayley Scales of Infant and Toddler Development, 3rd edition (Bayley-III). Cognitive and motor composite scores were used (mean in a normative population 100 , SD 15).

\section{Statistical Analysis}

Data analysis was performed using IBM SPSS Statistics (SPSS, Chicago, IL). Growth and nutrition details were related to volumes of the different brain structures (BGT, CGM, cerebellum, and TBV), FA-values in the PLIC, and neurodevelopmental outcome using linear regression analyses. First, brain volumes were adjusted for PMA at the time of scan. To this end, brain tissue volume-PMA ratios were calculated for each brain structure. FA values were corrected for PMA at the time of scan by calculating FA-PMA ratios. As illness severity is known to influence brain development, perinatal

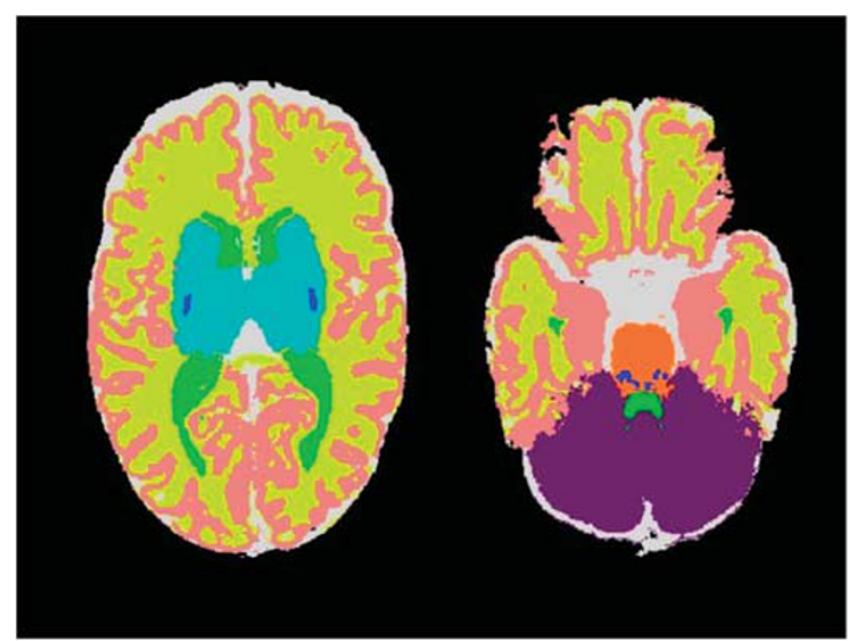

Figure 1. Example segmentation results. Representative example of segmentation data as obtained using the automatic segmentation method by Anbeek et al. (17). Different brain structures are depicted in different colors. Pink = cortical gray matter, yellow = unmyelinated white matter, light blue $=$ basal ganglia and thalami, dark blue $=$ myelinated white matter, purple $=$ cerebellum, orange $=$ brainstem, dark green $=$ cerebrospinal fluid (CSF) in the ventricles, gray $=$ CSF outside the brain.
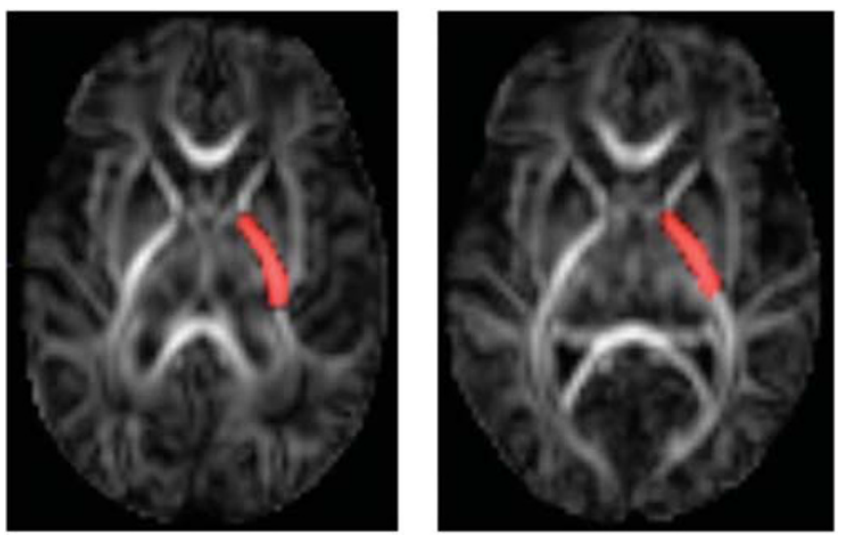

Figure 2. Example DTI data. Two representative examples of fractional anisotropy (FA)-map of DTI data after image-processing and template registration (see Methods section). Red label reflects the right PLIC, as labeled in the neonatal template by Oishi et al. (18). FA values were extracted from these labels (i.e., left and right PLIC). DTI, diffusion tensor imaging; FA, fractional anisotropy; PLIC, posterior limb of the internal capsule.

and postnatal risk factors were subsequently included as covariates in a multivariable regression model. The following variables were entered into the model: GA, birth weight (BW) $z$-score, computed according to the Dutch Perinatal registry reference data (20), gender, WMI score, bronchopulmonary dysplasia (BPD), patent ductus arteriosus (PDA), blood culture-proven sepsis, necrotizing enterocolitis (NEC), intraventricular hemorrhage (IVH), cerebellar injury, and duration of PN. In the final multivariable regression model, relevant perinatal and neonatal factors that showed an association with brain metrics and/or neurodevelopmental outcome at the alpha level $<0.05$ were included. Results were presented as coefficients of the independent variables with the $95 \%$ confidence intervals. 


\section{RESULTS}

The demographic and clinical characteristics of the 131 patients enrolled in the study are shown as mean, SD, and range in Table 1. Enteral intake and cumulative parenteral and enteral intakes in the first 4 weeks after birth are shown in Table 2 .

\section{Early Nutrition and Brain Volumes at TEA}

Overall results are shown in Table 3. After correction for BW $Z$-score, GA, PMA, WMI, and clinical risk factors (BPD, PDA, blood culture-proven sepsis, NEC, IVH, and cerebellar injury), cumulative fat and caloric intakes were positively related to larger cerebellar volumes $(P<0.01$ and $P<0.05$, respectively). Enteral protein, fat, and caloric intakes were also positively associated with cerebellar volume (all $P<0.01$; Figure 3). Increased cumulative fat, enteral protein, fat, and caloric intake were associated with larger BGT volumes (all $P<0.05)$. Enteral protein intake was related to larger TBV $(P=0.01)$. After correction for BW $Z$-score, GA, PMA, WMI, and clinical risk factors (PDA, NEC, BPD, sepsis, IVH, and cerebellar injury) longer duration of $\mathrm{PN}$ was associated with smaller TBV, cerebellar, BGT, and CGM volume (all $P<0.01)$. Conversely, weight gain, measured in $\mathrm{g} / \mathrm{kg} / \mathrm{day}$, during the first 4 weeks showed a significantly positive association with TBV, cerebellar, BGT, and CGM volumes (all $P<0.01$; Figure 4). Cumulative protein intake, the amount of $\mathrm{MM}, \mathrm{HC}$, and length growth were not related to any of the brain volume measurements.

\section{Early Nutrition and White Matter Maturation at TEA}

Cumulative caloric and fat intakes, after correction for GA, BW Z-score, gender, WMI, IVH, BPD, and PDA, were positively associated with FA in the left PLIC as measured at TEA (all $P<0.05$ ). Enteral protein, fat, and caloric intakes over the first 28 days of life showed a positive relationship with FA in the PLIC (all $P<0.01$; Figure 5), whereas days of PN showed a negative association with FA $(P<0.05)$. Weight gain, HC, and length growth in the first 4 weeks were not correlated with FA in the PLIC (Table 3). The amount of MM was not related to FA in the PLIC. A positive association between the amount of MM and FA in the left PLIC was found in males only $(B=0.001$, confidence interval $=0.000-$ $0.002, P<0.05)$.

\section{Early Nutrition and Neurodevelopmental Outcome at 2 Years' CA}

Cumulative protein intake was positively associated with higher cognitive and motor scores at 2 years' CA (all $P<0.05)$. Findings persisted after controlling for BW $Z$-score, $\mathrm{GA}$, gender, WMI, and clinical risk factors (BPD, PDA, blood culture-proven sepsis, NEC, and IVH; all $P<0.05)$. Cumulative energy intake showed a trend on cognitive score $(P=0.051)$. Enteral intake, amount of $M M$, weight gain, $\mathrm{HC}$, and length growth in the first 4 weeks were not related to neurodevelopmental outcome at 2 years' CA (Table 4 ).
Table 2. Feeding characteristics and nutritional intake

\begin{tabular}{|c|c|}
\hline $\begin{array}{l}\text { Nutritional details/feeding characteristics } \\
\text { and nutritional intake }\end{array}$ & $\begin{array}{l}\text { Mean and SD or } \\
N \text { (percentage) }\end{array}$ \\
\hline Parenteral nutrition (days) & $15 \pm 4$ \\
\hline Days until full enteral feeds & $15 \pm 4$ \\
\hline Weight gain $(\mathrm{g} / \mathrm{kg} / \text { day })^{\mathrm{a}}$ & $8 \pm 2$ \\
\hline Weight gain 1 week (g/kg/day) & $-5 \pm 10$ \\
\hline Weight gain 2, 3, 4 weeks (g/kg/day) & $14 \pm 5$ \\
\hline $\mathrm{HC}$ growth $\left(\mathrm{cm} /\right.$ weeks) ${ }^{\mathrm{a}}$ & $0.3 \pm 0.2$ \\
\hline Length $\left(\mathrm{cm} /\right.$ weeks) ${ }^{a}$ & $0.7 \pm 0.4$ \\
\hline Cumulative protein intake ${ }^{a}$ (g/kg/day) & $2.4 \pm 0.1$ \\
\hline Protein intake 1 week (g/kg/day) & $2 \pm 0.3$ \\
\hline Protein intake 2, 3, 4 weeks (g/kg/day) & $2.6 \pm 0.2$ \\
\hline Cumulative fat intake $^{\mathrm{a}}$ (g/kg/day) & $4.3 \pm 0.8$ \\
\hline Fat intake 1 week (kcal/kg) & $1.4 \pm 0.5$ \\
\hline Fat intake 2, 3, 4 weeks (g/kg/day) & $5.2 \pm 1.1$ \\
\hline Cumulative caloric intake ${ }^{\mathrm{a}}(\mathrm{kcal} / \mathrm{kg})$ & $95 \pm 11$ \\
\hline Caloric intake 1 week (kcal/kg) & $51 \pm 7$ \\
\hline Caloric intake 2, 3, 4 weeks (kcal/kg) & $110 \pm 14$ \\
\hline Protein from enteral nutrition $^{\mathrm{a}}$ ( $\left.\mathrm{g} / \mathrm{kg} / \mathrm{day}\right)$ & $1.5 \pm 0.4$ \\
\hline Fat from enteral nutrition $^{\mathrm{a}}$ ( $\mathrm{g} / \mathrm{kg} /$ day) & $3.8 \pm 1.0$ \\
\hline $\begin{array}{l}\text { Calories from enteral nutrition }{ }^{\mathrm{a}} \text { ( } \mathrm{kcal} / \mathrm{kg} / \\
\text { day) }\end{array}$ & $72 \pm 20$ \\
\hline
\end{tabular}

\section{DISCUSSION}

The results of this observational cohort study reveal early weight gain and macronutrient intake to be positively related to brain volumes of specific brain structures including the cerebellum and central gray matter, white matter maturation at TEA, and to neurodevelopmental outcome in late infancy in very preterm infants. These associations appeared to be independent of illness severity, as they persisted after adjustment for several variables reflecting neonatal morbidity.

\section{Early Nutrition and Brain Volumes at TEA}

The findings of a positive association between weight gain, nutritional intake, and volumes of different brain structures as observed in our study are in line with findings previously reported in the literature. Tan et al. found higher energy intake in the first 28 days to be related to larger TBVs and higher mental and psychomotor scores on the Bayley Scales of Infant and Toddler Development at 3 months of age (10).

Other studies in preterm infants that evaluated the influence of nutrition on brain size and neurodevelopmental outcome found contradictory results $(11,21,22)$. HansenPupp et al. did not find any correlation between protein and caloric intake and brain volumes at TEA, nor with neurodevelopmental outcome at 2 years' CA; however, the authors revealed higher insulin-like growth factor-1 levels to be associated with larger brain volumes of the unmyelinated white matter, gray matter, and cerebellum as measured at 
TEA $(11,21)$. In our study, nutritional intake displayed the strongest association with cerebellar volume. The cerebellum shows the highest growth rate compared with other intracranial structures during the last trimester of pregnancy. This phase of rapid cerebellar development likely represents a period of particular vulnerability. It has been demonstrated that preterm infants without any evidence of brain injury on their MRI showed smaller cerebellar volumes at TEA compared with term infants $(23,24)$. This finding suggests that cerebellar development is impeded during the early weeks of premature life, even in the absence of demonstrable cerebral or cerebellar MRI injury, and may therefore be particularly sensitive to environmental factors including nutrition $(23,25)$. Experimental studies support this. Maternal protein malnutrition in mice reduced cell proliferation and thickness in the external granular layer and reduced the number of calbindin-positive Purkinje cells and granular cells in the internal granular layer of the cerebellum in their offspring (26). Shorter duration of PN and higher enteral nutrition intake were related to larger volumes of the BGT as measured at TEA. These findings are in line with previous observations from a long-term follow-up study, in which standard preterm formula given during the first 4 weeks after birth in very-preterm infants was related to larger caudate volumes at the age of 16 years in comparison with standard full-term formula (22). Furthermore, larger caudate volumes were related to higher verbal IQ in males. Our results now suggest that beneficial effects of improved nutrition on brain development may manifest as early as TEA. In addition, GA and severity of respiratory distress/BPD were described to be associated with reduction in basal ganglia volume (27). In our study, nutrition and weight gain were associated with larger BGT volumes, which persisted after adjustment for BPD. On the basis of our findings we speculate that some of the adverse effects of severe respiratory disease might be mitigated by adequate nutritional support.

Duration of PN showed a negative impact on brain volumes and FA in the PLIC. An explanation for this observation may be that infants who need PN longer are generally sicker and frequently exhibit enteral nutrition intolerance. Macronutrient intakes from PN are often suboptimal because of the need of fluid restriction and supplemental infusions in critically ill infants, especially soon after birth. Furthermore, current amino-acid mixtures are not optimized for preterm infants with different metabolic conditions and at different stages of development. In the present study it is not possible to disentangle the effect of inadequate nutritional intake from the effect of severe illnesses. Additional analyses revealed that weight gain during first 4 weeks was associated with larger brain structures at TEA. Previous studies examining intrauterine growth retardation (IUGR) and brain volumes early in life showed that IUGR was associated with significant volume reductions in CGM volumes, hippocampus, and infratentorial brain regions (including the brainstem and cerebellum) (28). In addition, a recent study demonstrated that IUGR newborns showed altered brain metabolite profiles and brain structures 

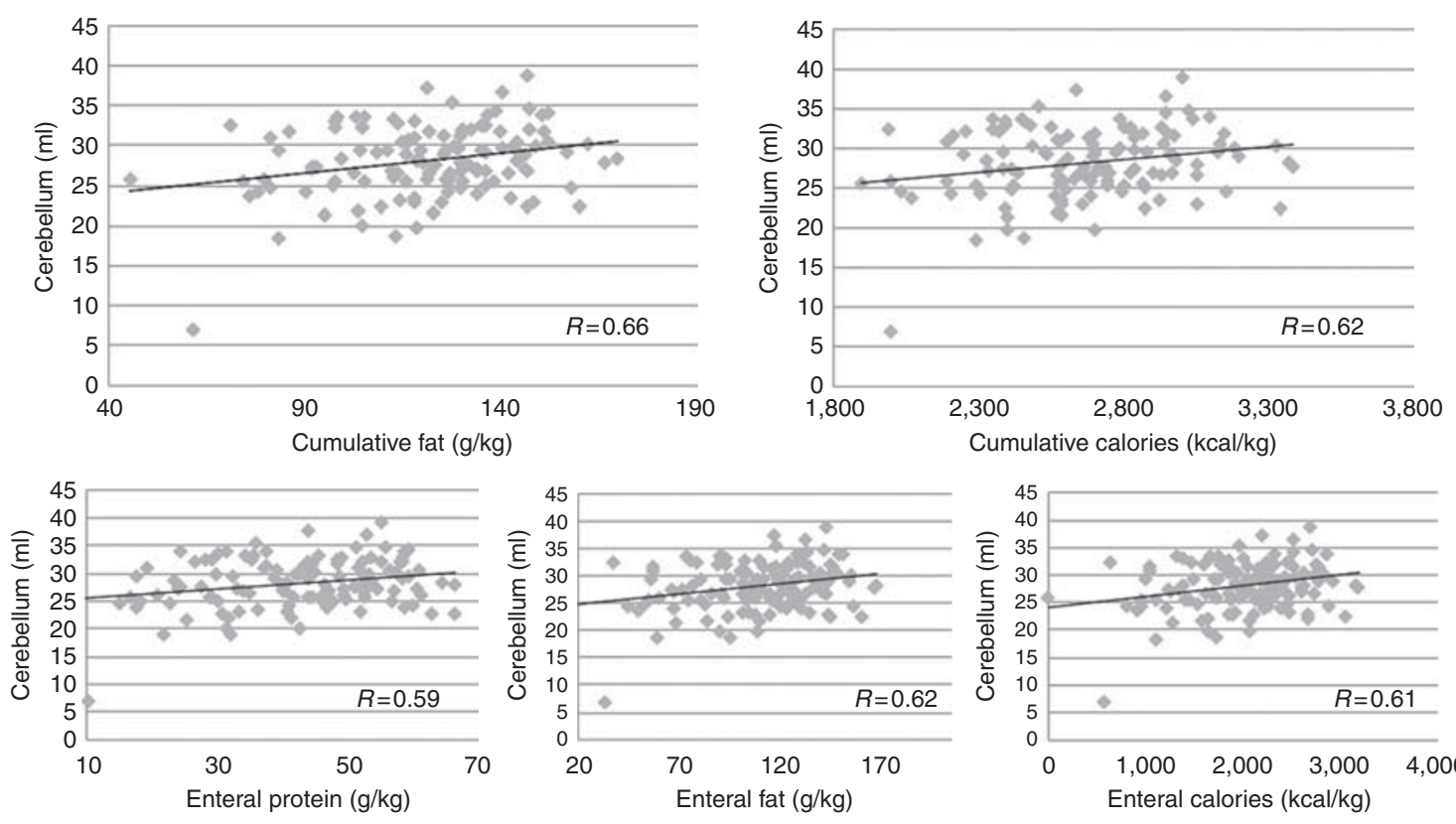

Figure 3. Association between nutrition intakes (cumulative and enteral) and cerebellar volume at TEA. TEA, term equivalent age.
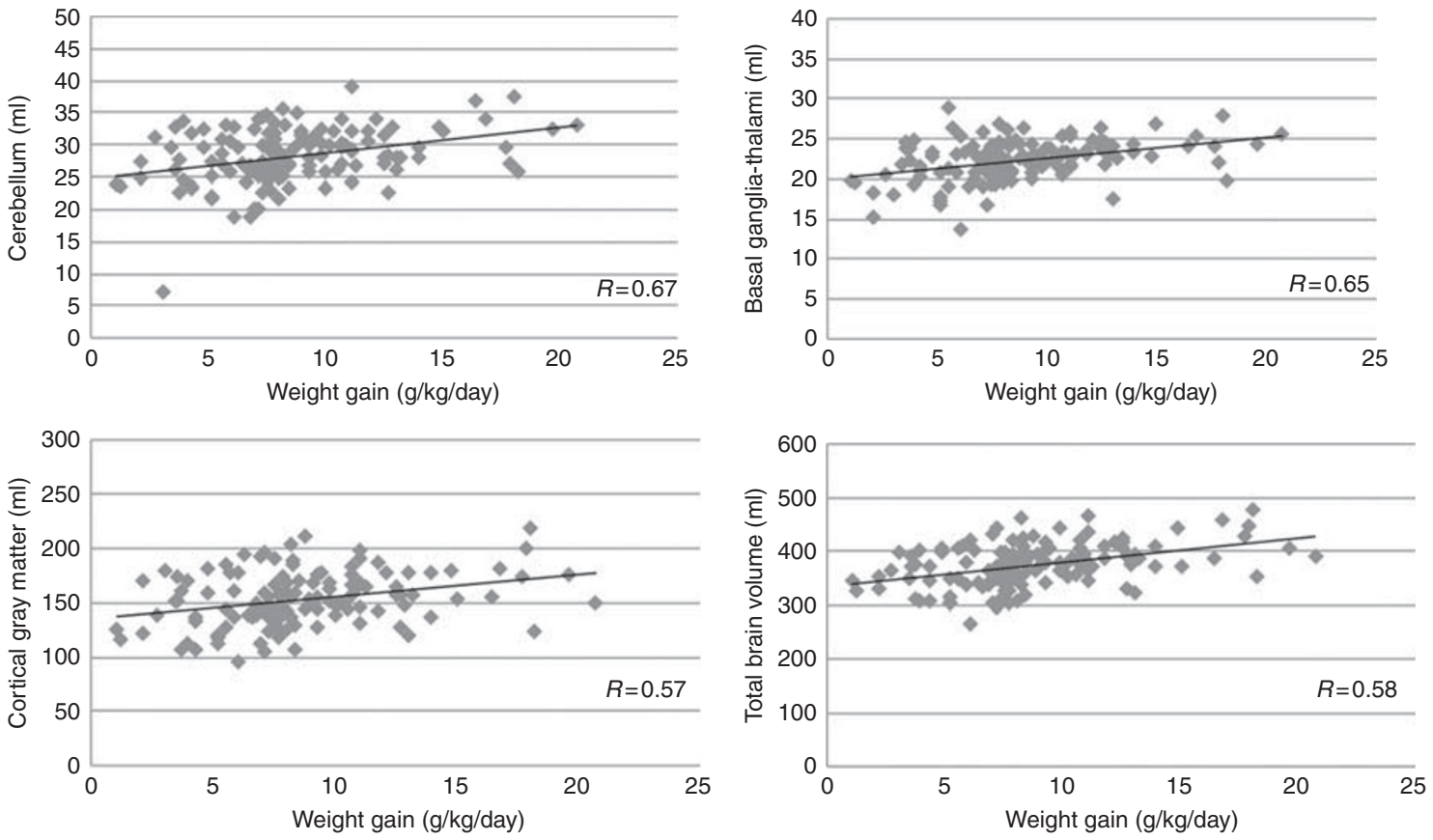

Figure 4. Association between weight gain, calculated as $\mathrm{g} / \mathrm{kg} / \mathrm{day}$, in the first 4 weeks after birth, and brain volumes at TEA. TEA, term equivalent age.

at 12 months of age $(29,30)$. The importance of postnatal growth for neurodevelopmental outcome of preterm infants has consistently been reported. Ehrenkranz et al. showed that faster weight gain and $\mathrm{HC}$ growth in the NICU were associated with higher cognitive and motor scores and a marked reduction in cerebral palsy at 18-22 months of age (2). Vinall et al. demonstrated a correlation between weight gain, HC growth, and cortical structural development in a DTI study (8).
Early Nutrition and White Matter Maturation at TEA

This study showed that increased enteral nutritional intake and shorter duration of $\mathrm{PN}$ were related to higher FA values in the PLIC as a measure of white matter maturation. FA in the PLIC has been shown to be positively related to increasing GA and better motor performance at 2 years' CA (31). Increase in FA observed with increasing maturation is thought to result from a combination of factors related to brain development, including an increase in myelination. 


\section{Articles | Coviello et al.}
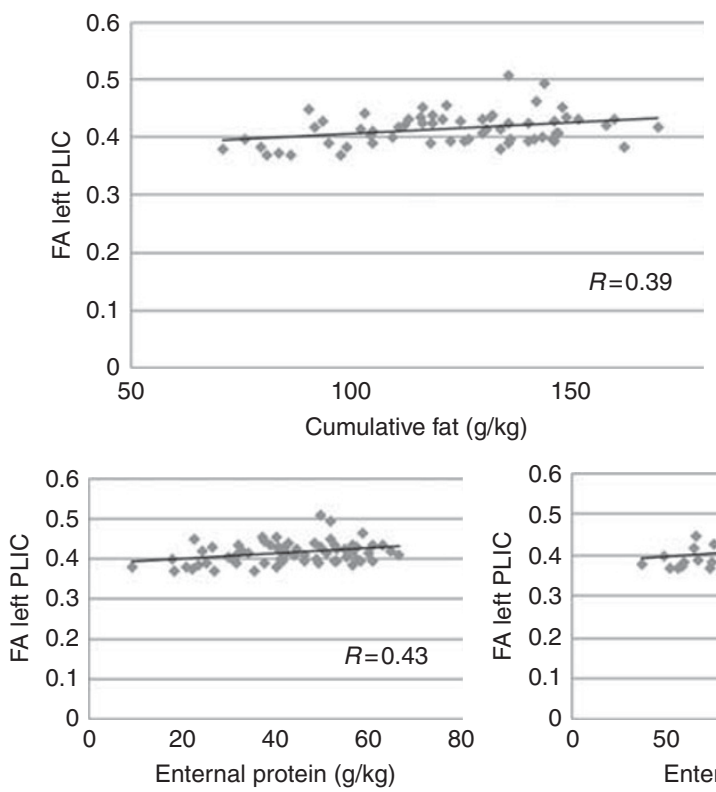
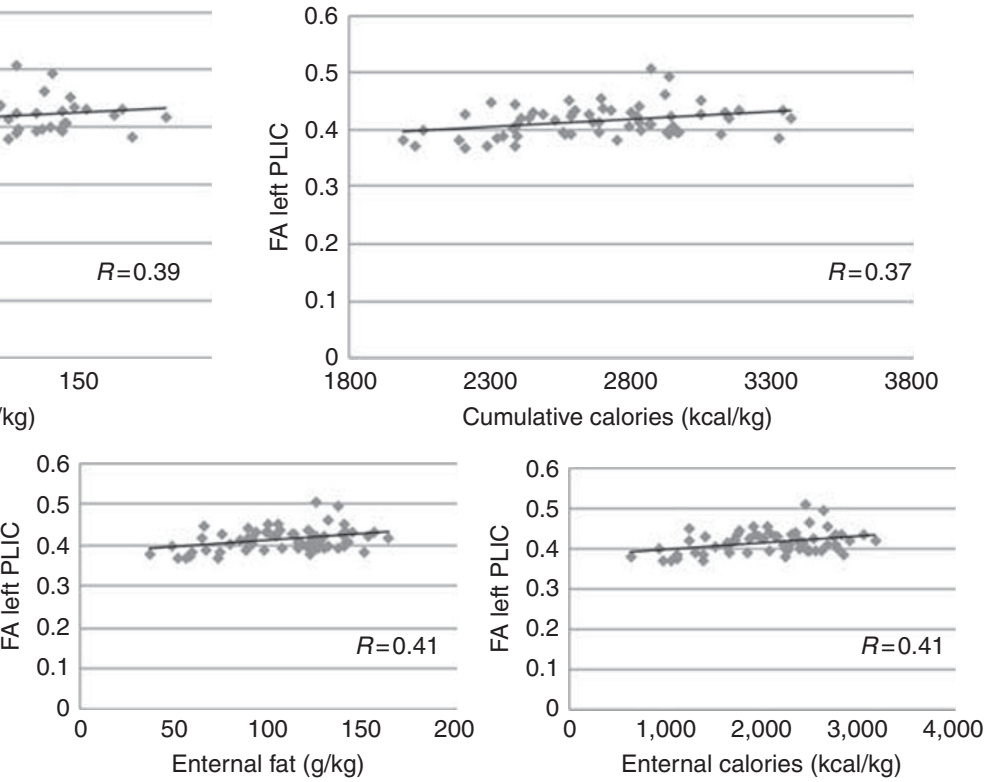

Figure 5. Association between nutrition intakes (cumulative and enteral) and FA values in the PLIC. FA, fractional anisotropy; PLIC, posterior limb of internal capsule.

Table 4. Multivariate linear regression between nutrition intakes (cumulative and enteral), postnatal growth, and neurodevelopment at 2 years' CA

\begin{tabular}{lcr}
\hline & Cognitive score; B (95\% Cl) & Motor score; B (95\% Cl) \\
\hline Cumulative protein $(\mathrm{g} / \mathrm{kg})$ & $0.51(-0.12-1.01)^{*}$ & $0.46(0.08-0.84)^{*}$ \\
Cumulative fat $(\mathrm{g} / \mathrm{kg})$ & $0.10(-0.01-0.22)$ & $0.03(-0.07-0.14)$ \\
Cumulative calories $(\mathrm{kcal} / \mathrm{kg})$ & $0.01(0.04-0.02)$ & $0.005(-0.003-0.012)$ \\
Weight gain $(\mathrm{g} / \mathrm{kg} / \mathrm{die})$ & $0.01(-0.08-0.1)$ & $-0.009(-0.09-0.78)$ \\
$\mathrm{HC}$ growth $(\mathrm{cm} / \mathrm{weeks})$ & $4.7(3.3-12.9)$ & $7.9(0.6-15.2)$ \\
Length $(\mathrm{cm} /$ weeks) & $4.1(1.1-9.3)$ & $2.6(1.8-7.2)$ \\
Enteral protein $(\mathrm{g} / \mathrm{kg})$ & $0.23(-0.20-0.49)$ & $0.08(-0.09-0.25)$ \\
Enteral fat $(\mathrm{g} / \mathrm{kg})$ & $0.10(-0.007-0.22)$ & $0.01(-0.06-0.10)$ \\
Enteral calories $(\mathrm{kcal} / \mathrm{kg})$ & $0.005(0.001-0.010)$ & $0.001(-0.003-0.005)$ \\
\hline
\end{tabular}

$\mathrm{CA}$, corrected age; $\mathrm{Cl}$, confidence interval; $\mathrm{HC}$, head circumference. ${ }^{*} P<0.05$.

According to our results, lipids seem particularly important for white matter maturation, showing a large effect size. Phospholipids such as arachidonic acid and docosahexaenoic acid (DHA) are essential for brain growth, function, and integrity, as they constitute $\sim 25 \%$ of the white matter. DHA incorporates into the membrane bilayer, and increases the degree of flexibility and direct interaction with membrane proteins. This has an impact on the speed of signal transduction and neurotransmission and might be related to brain growth and development (32). A recent study in 14 preterm infants, who received an enhanced parenteral and enteral feeding regimen, reported improved $\mathrm{HC}$ and decreased regional white matter diffusivity at TEA, the latter being suggestive of improved maturation of cerebral white matter tracts (33).

In addition, Dabydeen et al. showed that WMI may be alleviated by nutritional intervention. In their study of 16 infants with perinatal brain damage, a high energy and protein diet during the first year after birth resulted in significantly larger axonal diameters of the corticospinal tract and $\mathrm{HC}$ at the age of 1 year (34). Our results are in line with these findings, suggesting that improved early nutrition may enhance white matter maturation.

A positive correlation between the amount of $\mathrm{MM}$ and FA in the left PLIC was found in males only. The mechanism by which $\mathrm{MM}$ is thought to exert its effects on cognitive development is mainly related to its fatty-acid composition, which contains DHA and trophic factors such as insulin-like growth factor-1. A recent study demonstrated that breastfeeding during infancy was associated with better white matter development at 8 years of age in boys (35). Multiple white matter regions in the left hemisphere displayed significantly higher FA values (35). Isaacs et al. showed that the percentage of MM that infants received in the neonatal period positively 
correlates with white matter volume and TBV and cognitive scores in boys in adolescence (36).

\section{Early Nutrition and Neurodevelopmental Outcome at 2 Years' CA}

In addition, our results showed a positive association between cumulative protein intake and neurodevelopment. Animal models have shown that adequate provision of protein during fetal development and the early postnatal period are essential for brain growth and myelination, and that the cortex, hippocampus, and cerebellum appear to be the most vulnerable brain structures to protein deficit (37). In humans, it has been demonstrated that optimizing protein and energy intake in the neonatal period has an impact on cognition, with effects persisting until adolescence $(38,39,22)$.

It is important to note that during the study period protein and lipid intakes were below current recommendations. This may limit the ability to apply these results to premature infants who are receiving adequate amounts of nutrients at other centers. Since 2013, a new feeding regimen that aimed to reach $3.5-4 \mathrm{~g} / \mathrm{kg} /$ day of protein and $3-4 \mathrm{~g} / \mathrm{kg} /$ day of lipid in the first days after birth has been applied in the Wilhelmina Children's Hospital. Whether increasing protein and lipid intake during this period will improve brain volumes and white matter maturation should be investigated.

\section{Limitations}

This study has some limitations that need to be taken into account. First, its retrospective and observational design may lead to the risk of confounding bias. In fact, the patient's feeding management could have been influenced by the physician's impression of the infant's health. Thus, enteral feedings might be initiated earlier and advanced faster in newborns supposed to be healthier. Second, the exact composition of MM was not measured and was therefore obtained from the literature. Significant interindividual variations on human milk composition have been reported, depending on the stage of lactation. Third, this cohort had a rather high blood culture-proven sepsis rate of almost $40 \%$. This might limit the ability to generalize these data to other centers. Furthermore, future research would benefit from extending specific infant growth parameters, such as body composition and nutrition measurements, to include data about neonatal gut microbiome composition. A growing literature draws attention to the importance of balanced gut microbiota for healthy early brain development and to promote cognitive functions $(12,40)$. The microbiome interacts with the brain through hormonal, immunological, and neural pathways. Given that inflammation has a critical role in white matter deficits in preterm infants, microbiota changes have been postulated to specifically influence growth and development of white matter structures (12).

\section{CONCLUSION}

In conclusion, the results of this retrospective cohort study reveal a consistent positive association between early postnatal nutritional intake in preterm infants and brain volumes at TEA of the cerebellum and BG. They also demonstrate a positive relationship of early-life nutritional intake with white matter maturation as reflected by higher FA in the PLIC and with neurodevelopmental outcome at the age of 2 years. Improved weight gain was related to larger brain volumes. These findings emphasize the importance of early postnatal growth and nutrition, with a balanced protein, fat, and caloric content for early brain development.

\section{STATEMENT OF FINANCIAL SUPPORT}

K.K. is supported by a grant from the Wilhelmina Children's Hospital Research Fund ("Vrienden van het WKZ") to M.P.v.d.H., and M.J.N.L.B. The research of A.L. is supported by VIDI Grant 639.072.411 from the Netherlands Organisation for Scientific Research (NWO).

\section{DISCLAIMER}

These sponsors had no influence on the study design, the collection, analysis, and interpretation of data, the writing of the report or the decision to submit the paper for publication.

Disclosure: The authors declare no conflict of interest.

\section{REFERENCES}

1. Ehrenkranz RA, Younes N, Lemons JA, et al. Longitudinal growth of hospitalized very low birth weight infants. Pediatrics 1999;104:280-9.

2. Ehrenkranz RA, Dusick AM, Vohr BR, Wright LL, Wrage LA, Kenneth Poole W. Growth in the neonatal intensive care unit influences neurodevelopmental and growth outcomes of extremely low birth weight infants for the National Institutes of Child Health and Human Development Neonatal Research Network. Pediatrics 2006;117:1253-61.

3. Embleton NE, Pang N, Cooke RJ. Postnatal malnutrition and growth retardation: an inevitable consequence of current recommendations in preterm infants? Pediatrics 2001;107:270-3.

4. Fuglestad A, Rao R, Georgieff MK, Code MM. The role of nutrition in cognitive development. In: Nelson, CA Luciana, M, eds. Handbook of Developmental Cognitive Neuroscience, 2nd edn. The MIT Press: Cambridge, MA, 2008, pp 623-42.

5. Moeskops P, Viergever MA, Mendrik AM, De Vries LS, Benders MJNL, Isgum I. Automatic segmentation of MR brain images with a convolutional neural network. IEEE Trans Med Imaging. 2016;35:1252-61.

6. Kersbergen KJ, Makropoulos A, Aljabar P, et al. Longitudinal regional brain development and clinical risk factors in extremely preterm infants. J Pediatr 2016;178:93-100.e6.

7. Volpe JJ. Brain injury in premature infants: a complex amalgam of destructive and developmental disturbances. Lancet Neurol 2009;8: $110-24$.

8. Vinall J, Grunau RE, Brant R, et al. Slower postnatal growth is associated with delayed cerebral cortical maturation in preterm newborns. Sci Transl Med 2013;5:168ra8.

9. Beauport L, Schneider J, Faouzi M, et al. Impact of early nutritional intake on preterm brain: a magnetic resonance imaging study lydie. J Pediatr 2016;181:29-36.e1.

10. Tan M, Abernethy L, Cooke R. Improving head growth in preterm infants-a randomised controlled trial II: MRI and developmental outcomes in the first year. Arch Dis Child Fetal Neonatal Ed 2008;93: F342-6.

11. Hansen-Pupp I, Hövel H, Löfqvist C, et al. Circulatory insulin-like growth factor-I and brain volumes in relation to neurodevelopmental outcome in very preterm infants. Pediatr Res 2013;74:564-9.

12. Goyal MS, Venkatesh S, Milbrandt J, Gordon JI, Raichle ME. Feeding the brain and nurturing the mind: linking nutrition and the gut microbiota to brain development. Proc Natl Acad Sci USA 2015;112: 14105-2. 


\section{Articles | Coviello et al.}

13. Patel A, Engstrom J, Meier P, Jegier B, Kimura R. Calculating postnatal growth velocity in very low birth weight (VLBW) premature infants. J Perinatol 2009;29:618-22.

14. Schanler R, Atkinson S Human milk. In: Tsang RC, Uauy R, Koletzko B, Zlotkin SH, eds. Nutrition of the Preterm Infant, 2nd edn. Cincinnati, OH: Digital Educational Publishing, 2005:333-56.

15. Woodward LJ, Anderson PJ, Austin NC, Howard K, Sc. B, Inder TE. Neonatal MRI to predict neurodevelopmental outcomes in preterm infants. N Engl J Med 2006;17:685-94.

16. Kidokoro H, Anderson PJ, Doyle LW, Woodward LJ, Neil JJ, Inder TE. Brain injury and altered brain growth in preterm infants: predictors and prognosis. Pediatrics 2014;134:E444-53.

17. Anbeek P, Išgum I, Van Kooij BJM, et al. Automatic segmentation of eight tissue classes in neonatal brain MRI. PLoS ONE 2013;8:1-9.

18. Oishi K, Mori S, Donohue PK, et al. Multi-contrast human neonatal brain atlas: application to normal neonate development analysis kenichi. Neuroimage 2012;56:8-20.

19. Klein S, Staring M, Murphy K, Viergever MA, Pluim JPW. elastix : a toolbox for intensity-based medical image registration. IEEE Trans Med Imaging 2010;29:196-205.

20. Visser GHA, Eilers PHC, Elferink-Stinkens PM, Merkus HMWM, Wit JM. New Dutch reference curves for birthweight by gestational age. Early Hum Dev 2009;85:737-44.

21. Hansen-Pupp I, Hövel H, Hellström A, et al. Postnatal decrease in circulating insulin-like growth factor-I and low brain volumes in very preterm infants. J Clin Endocrinol Metab 2011;96:1129-35.

22. Isaacs EB, Gadian DG, Sabatini S, et al. The effect of early human diet on caudate volumes and IQ. Pediatr Res 2008;63:308-14.

23. Van Kooij BJM, Benders MJNL, Anbeek P, Van Haastert IC, De Vries LS, Groenendaal F. Cerebellar volume and proton magnetic resonance spectroscopy at term, and neurodevelopment at 2years of age in preterm infants. Dev Med Child Neurol 2012;54:260-6.

24. Limperopoulos C, Soul JS, Gauvreau K, et al. late gestation cerebellar growth is rapid and impeded by premature birth. Pediatrics 2005;115: 688-95.

25. Volpe JJ. Cerebellum of the premature infant: rapidly developing, vulnerable, clinically important. J Child Neurol 2009;24:1085-4.

26. Ranade SC, Sarfaraz Nawaz M, Kumar Rambtla P, Rose AJ, Gressens P, Mani S. Early protein malnutrition disrupts cerebellar development and impairs motor coordination. Br J Nutr 2012;107:1167-75.

27. Inder TE, Warfield SK, Wang H, Hüppi PS, Volpe JJ. Abnormal cerebral structure is present at term in premature infants. Pediatrics 2005;115: 286-94.
28. Tolsa CB, Zimine S, Warfield SK, et al. Early alteration of structural and functional brain development in premature infants born with intrauterine growth restriction. Pediatr Res 2004;56:132-8.

29. Simões RV, Muñoz-moreno E, Cruz-lemini M, Eixarch E, Sanz-cortes M, Gratacós E. Brain metabolite alterations in infants born preterm with intrauterine growth restriction: association with structural changes and neurodevelopmental outcome. Am J Obstet Gynecol 2016;216: 62.e1-14.

30. Padilla N, Junqué C, Figueras F, et al. Differential vulnerability of gray matter and white matter to intrauterine growth restriction in preterm infants at 12 months corrected age. Brain Res 2014;1545:1-11.

31. Van Kooij BJM, De Vries LS, Ball G, et al. Neonatal tract-based spatial statistics findings and outcome in preterm infants. Am J Neuroradiol 2012;33:188-94.

32. Vanhatalo S, Kaila K. Development of neonatal EEG activity: from phenomenology to physiology. Semin Fetal Neonatal Med 2006;11: 471-8.

33. Strommen K, Blakstad EW, Moltu SJ, et al. Enhanced nutrient supply to very low birth weight infants is associated with improved white matter maturation and head growth. Neonatology 2015;107:68-75.

34. Dabydeen L, Thomas JE, Aston TJ, Hartley H, Sinha SK, Eyre JA. Highenergy and -protein diet increases brain and corticospinal tract growth in term and preterm infants after perinatal brain injury. Pediatrics 2008; 121:148-56.

35. Ou X, Andres A, Cleves MA, et al. Sex-specific association between infant diet and white matter integrity in 8-y-old children. Pediatr Res 2014;76: 535-43.

36. Isaacs EB, Fischl BR, Quinn BT, Chong WK, Gadian DG, Lucas A. Impact of breast milk on IQ, brain size and white matter development. Pediatr Res 2010;67:357-62.

37. Georgieff MK. Nutrition and the developing brain: nutrient priorities and. Am J Clin Nutr 2007;85:614-20.

38. Lucas A, Morley R, Cole T, et al. Early diet in preterm babies and developmental status at 18 months. Lancet 1990;335:1477-81.

39. Stephens BE, Walden RV, Gargus RA, et al. First-week protein and energy intakes are associated with 18-month developmental outcomes in extremely low birth weight infants. Pediatrics 2009;123:1337-43.

40. Keunen K, van Elburg R, van Bel F, Benders M. Impact of nutrition on brain development and its neuroprotective implications following preterm birth. Pediatr Res 2015;77:148-55.

41. Papile LA, Burstein J, Burstein R, Koffler H. Incidence and evolution of subependymal and intraventricular hemorrhage: a study of infants with birth weights less than 1,500 gm. J Pediatr 1978;92:529-534. 